NISTIR 89-3929

\title{
PLANAR NEAR-FIELD CODES FOR PERSONAL COMPUTERS
}

Lorant A. Muth

Richard L. Lewis

National Institute of Standards and Technology U.S. Department of Commerce

Boulder, Colorado 80303-3328

October 1989 


\section{PLANAR NEAR-FIELD CODES FOR PERSONAL COMPUTERS}

Lorant A. Muth

Richard L. Lewis

Electromagnetic Fields Division

Center for Electronics and Electrical Engineering

National Engineering Laboratory

National Institute of Standards and Technology

Boulder, Colorado 80303-3328

October 1989

Sponsored, in part, by

Air Force Guidance and Metrology Center

Newark Air Force Base, Ohio 43057

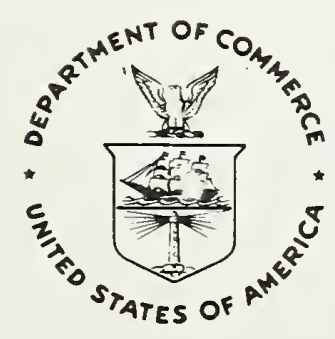



Abstract . . . . . . . . . . . . . . . . 1

1. Introduction . . . . . . . . . . . . . . . 1

2. General Features . . . . . . . . . . . . . 2

3. Data Management . . . . . . . . . . . . . . 4

4. Research Modules . . . . . . . . . . . . . . . . 6

5. Output Files . . . . . . . . . . . . . . . . 10

6. DOS Batch Files . . . . . . . . . . . . . . . 11

7. Symbol Definitions . . . . . . . . . . . . . . 12

8. Subroutine Descriptors . . . . . . . . . . . . . . . 12

9. Table of Dependencies . . . . . . . . . . . . . . . . 13

10. Summary . . . . . . . . . . . . . . . . . 13

Appendix A . . . . . . . . . . . . . . . . . . . 35

Appendix B . . . . . . . . . . . . . . . . . . 36

Appendix C . . . . . . . . . . . . . . . . . 38

Appendix D . . . . . . . . . . . . . . . . . . 41 


\section{•}




\section{Planar Near-Field Codes for Personal Computers \\ by}

Lorant A. Muth and Richard L. Lewis

We have developed planar near-field codes, written in Fortran, to serve as a research tool in antenna metrology. We describe some of the inner workings of the codes, the data management schemes, and the structure of the input/output sections to enable scientists and programmers to use these codes effectively. The structure of the codes is seen to be open, so that a user can incorporate a new application into the package for future use with relative ease. The large number of subroutines currently in existence are briefly described, and a table showing the interdependence among these subroutines is constructed. Some basic research problems, such as transformation of a near field to the far field and probe position error correction, are carried out from start to finish, to illustrate use and effectiveness of these codes. Sample outputs are shown. The advantage of a high degree of modularization is demonstrated by the use of DOS batch files to execute Fortran modules in a desired sequence.

Key words: antenna metrology; data management; planar near-field codes; research tool; subroutines

\section{Introduction}

Most research problems in antenna metrology are computationally intensive, and program development makes up a substantial part of the research effort. Hence, isolating frequent computational themes in this research area and developing independent modules that can perform any of these computational themes in any order independently of any previous computational step is very desirable. Improvements in both the quality and quantity of research can be a by-product of such a computational tool. Ideally, such a software package should be an open-ended system; that is, new modules can be added to it painlessly to increase the versatility of the package. It should also be easy to use and learn, and, therefore, adaptable to new areas of research. With the cooperative effort of the members of the Antenna Metrology Group such a software package could evolve into a comprehensive research tool over a short period of time.

With these thoughts in mind, we have taken the first steps to accomplish the goal of creating a comprehensive software package suitable for conducting state-ofthe-art research on a personal computer. ${ }^{1}$ We have achieved a very high level of modularity by creating a large number of subroutines (written in Fortran) that can

1 Because most antenna metrology research problems are computationally intensive and usually have large memory requirements, the authors recommend that a personal computer equipped with the fastest available CPU and floating point processor be used and that at least 4 megabytes of RAM be made available. 
be used in many different contexts, since the subroutines emphasize structure rather than content of small computational problems. By the same effort, we have made it relatively simple to create higher level subroutines, since such routines can rely heavily on the existing low level subroutines of general applicability. These higher level routines accomplish more complicated and complete computational tasks than the low level subroutines. In turn, they can be combined to form independent modules, which are the selected subtasks of a particular research effort. These subtasks usually will be subtasks in other research areas, too. Hence, the effort expended in creating them will be saved many times over in future endeavors.

Particular attention has been given to the way information flows to and from the modules and between modules. We have automated much of the data management needed to provide a smooth transition as one module finishes its task and another is executed to accomplish the next step of the research. A large number of small modules, playing a supportive role in data management, have been created to allow manipulation of datasets according to the needs of the current phase of the research project. For example, an existing dataset merely has to be activated to make it accessible to a module about to be executed. Thus, both the modules chosen to be executed and the datasets to be used can be controlled interactively by the scientist. This makes for a very flexible computational procedure, freeing one's time and energy to think about research procedure rather than computational detail.

In the next section, concentrating on the main features, we outline the structure of the Planar Near-Field Codes (PNFC), and in the subsequent sections we present essential details of the main features. It is our intention that any researcher, programmer or scientist, be able to use these codes effectively after familiarizing himself with the contents of this report.

\section{General Features}

The complete PNFC is structured into modules. To be able to determine the function of a module we merely have to decipher the acronym that was constructed to name the module. Once deciphered, the full function of the module should be self-evident. In Table 1 we have compiled the symbols used to construct module names and define the meaning of each symbol. In Table 2 and 3 we list the modules used to conduct research and the modules used to manage data access during the course of research, respectively. A brief descriptor of their function is also included.

All of the research modules listed in Table 2 perform some manipulation of an existing dataset, that is, they either numerically transform the dataset or perform some I/O operation on it. Each module was designed to perform a single computational task that is an important aspect of research in antenna metrology. Some of the modules are more specific to antenna metrology than others. For example, the module URDNFFF (Utility, ReaD a Near Field and transform it to the Far Field) is an ever present computational step in this research area, but UPRNCBD (Utility, PRiNt a Complex Binary Dataset) is obviously of more general applicability. How to execute these modules is demonstrated in Section 4.

The modules listed in Table 3 perform simple data management functions. For example, USWTOFF (Utility, SWitch TO Far Fields) activates the far field datasets 
that have been previously created and recorded within the data management part of the system. After USWTOFF has been executed any subsequent executions of modules that can use either far-field or near-field data will access the far-field datasets, unless this switch is overridden by a nonzero active field. How to activate a specific dataset to make it the dataset that any module will use will be covered in Section 3.

All these modules are constructed from a large set of independent subroutines that perform specific computational or I/O subtasks. They are used repeatedly in various sequences to produce the specific results of the module. These subroutines are compiled into a library, which is linked to a module at compilation time. All existing subroutines are listed in Table 7, along with a brief description of their function.

All modules use a general set of input parameters that belong to the original dataset. The original datasets are recorded as direct access binary files, so that specific records within them can be accessed at will. This might be useful if some selected records are found to be in error. (How to create the original direct access datasets from some ascii file that was created on some other computer or data acquisition system is explained in Appendix A.) The first 7 records in these datasets contain the essential parameters of the dataset. All modules access the original direct access file to input the original parameters of the dataset, although only a subset of these might actually be needed by the specific module in use. This procedure assures that the same parameter set will be used by all modules for a specific dataset. A list of these parameters is given in Appendix $A_{i}$

Each module might also access a parameter (.PAR) file that is specific to it. For example, UMAKEDZ (Utility, MAKE DZ), which creates a probe displacement error function, reads the parameter file PERDZ.PAR if periodic error functions are requested, and UTS (Utility, Taylor Series) reads the parameter file SCALE.DZ to input the amplitude of the error function requested for the current execution. The parameter files currently in existence and the research modules that access them are listed in Table 4. The parameter files accessed by the data management modules are tabulated in Table 5. The contents of each parameter file will be defined in Section 3.

All necessary I/O procedures are handled within each module, but there are some specific modules that prepare the data and create ascii files that can be further processed for graphical output. Two such modules are UCBDGRD (Utility, Complex Binary Dataset to .GRD file) and UCBDDAT (Utility, Complex Binary Dataset to .DAT file), which create ascii datasets to be used for plotting 3-D and simple linear plots, respectively. These modules also rely on specific parameter files to perform their function as desired. These parameter files are listed in Table 4.

Finally, all modules have very similar structures and differ significantly only in their computational sections. The common structure is as follows:

a. Read all relevant switch settings and determine the unit numbers ${ }^{2}$ of existing

2 Here and throughout this report unit numbers refer to the file extensions $x x$ in 
datasets. Check to see whether there is room for more datasets on the disk and assign the new unit numbers.

b. Read all relevant parameters needed by the module.

c. Read all parameters describing the dataset to be used.

d. Read all datasets needed by the module.

e. Prepare for computations.

f. Perform the computations.

g. Output the results to the preassigned units.

h. Set the relevant switches and update the unit numbers of the new datasets.

i. Output an ascii file to record all parameters and I/O activity.

j. Update the history file to show which modules were executed.

k. Stop execution of the module with 'Successful termination' message.

This structure seems to be very successful in that modules that are truly independent of each other have been constructed, which, therefore, can be executed in any order as long as the relevant datasets have been created. Under these conditions a research project can be implemented with relative ease either interactively or with the use of DOS batch files. (The use of DOS batch files to enhance research efficiency is discussed in Section 6.)

\section{Data Management}

In this section we present the details of unit or dataset management built into the system as a whole. Specific modules make use of this procedure according to their requirements. Here the terms data management and unit management have the same meaning, as datasets generated by PNFC for the purpose of computations reside on files with filenames FORT. $x x$, where $x x$ is some integer refering to a Fortran unit number assigned internally by the module being executed. (The filename FORT is automatically assigned when a Fortran binary write is executed.)

a. Initialization of the system.

The system has to be initialized before starting any research project with a new dataset. Both the system parameters and the unit numbers where different datasets will reside are initialized in this procedure. Here we will describe how the unit numbers are set and manipulated at the start of the research project. In Appendix B the output of the initialization module is shown and an explanation of features not covered in this section is presented.

When the UINITUN (Utility, INITialize Unit Numbers) module is executed the initial unit numbers for the far-field and the near-field datasets are read from a parameter file (INIT.IUN) and entered into the unit number files named FF.IUN and NF.IUN. After initialization the modules URDFFNF or URDNFFF can be executed to read in the existing direct-access complex binary dataset containing the original data to be analyzed. (Subsequently, the same modules will access datasets according to the unit management switch settings. See Section $3 \mathrm{~b}$ below.)

the filenames fort.xx that are automatically generated by the system when a binary dataset is written to the disk. 
Both modules output both the far field and the near field to FORT.xx files; the filename extensions $x x$ are obtaqined from the files FF.IUN and NF.IUN.

All far fields created after initialization will be assigned unit numbers one less than the previously assigned far field unit number, and all near fields created after initialization will be assigned unit numbers one higher than the previously assigned near field unit number. Hence, the far-field and near-field unit numbers will converge toward each other as datasets are created by executing module after module. Before any module proceeds with execution of its task it checks to see whether there is enough of a difference between the last far-field and the last near-field unit numbers to allow the creation of additional datasets. If the far-field and near-field unit numbers are adjacent to each other, no module that creates a new dataset is allowed to proceed, and an appropriate error message to that effect is displayed. In this manner, disk overload is prevented, since new datasets cannot be created indefinitely.

b. The Complex Binary Dataset (CBD) files.

Except for the original datasets, which are stored as direct access binary files, the modules read and write complex binary datasets (CBD) during execution to store intermediate results in the course of the research project. These datasets are recorded with the filename FORT and with integer unit numbers for extensions. The unit numbers are automatically assigned, as described in the previous section. For example, FORT.40 would be the initial near field unformatted complex binary file, and FORT.60 would be the initial far field unformatted complex binary file.

Since all modules read and/or write one or more CBD files, we must keep track of these files and must be able to access a desired dateset with relative ease. For this purpose a unit number management support system has been constructed. This works as follows:

An existing dataset is identified by its unit number, which is the extension of the FORT file. An existing unit number is any unit number that has been created since initialization. An existing unit number, in general, has no special status and is not automatically accessed by any module until it is made active, additional, or current. A unit number is active if its value is recorded in the ACTIVE.IUN file, whereas a unit number is additional if its value is recorded in the ADD.IUN file. The current unit numbers are the last unit numbers recorded in the files FF.IUN and NF.IUN. In general, these are the unit numbers created by the most recently executed module, but can be altered according to the user's needs. A general purpose module can access either the current near field unit number or the current far field unit number, depending on the setting of the variable FFNF recorded in the file FFORNF.IUN. The variable FFNF can have the values 'ff' or 'nf'.

When modules access datasets a precedence rule is followed: the ACTive file gets accessed first, and the ADDitional file gets accessed if the module requires 2 datasets. The current file gets accessed only if the ACTive file is set to 0, and any existing file can be accessed only if it is made ACTive, ADDitional or current. To access the desired current files with modu'es that process either far-field or nearfield datasets the 'FFORNF' switch has to be set to tell the system that one is 
interested in far-field or near-field unit numbers.

A number of utilities have been written to define these file types easily. These utilities are listed in Table 3. To view the existing unit numbers we execute USHOWUN (Utility, SHOW Unit Numbers), which summarizes the existing files according to their type (as defined in FFORNF.IUN) and status (ACT, ADD, current, existing). USHOWUN will also identify the unit numbers of special datasets, such as the TS (Taylor Series) file, EC (error corrected) and DS (direct sum) files. To activate a dataset, execute one of the special utilities listed in Table 3. Similarly, we can $a d d$ a dataset. To make a dataset current, one can execute the decrementing or incrementing modules (UDECFF, UDECNF, UINCFF, UINCNF) repeatedly until the desired unit number is the last unit number shown by USHOWUN. Two examples of the output of USHOWUN are given in Appendix $\mathrm{C}$ along with explanations.

\section{c. Output Files.}

As discussed above most modules read and write CBD files according to the unit management scheme built into every module. In addition, some of the modules create special ascii files to be used as input to graphics programs. The module UCBDGRD, for example, reads the ACTive or current CBD file, with filename FORT and an extension defined by the active or current unit number. It then outputs ascii files, whose filenames are obtained by concatenating the setting of the switch FFORNF with the descriptors AMP or PHASE, and appending a filename extension .GRD. The structure of these files is determined by the requirement of the graphics package in use. Similary, the module UCBDDAT creates ascii files for simple $x y$-plots with filenames obtained the same way as for .GRD files, using .DAT as the filename extension. This module outputs a set of $x$-values and one, two or three $y$-values. The actual number of data columns output by UCBDDAT is determined by the ACTive, ADDitional and current switch settings. The rules are as follows: to write only a single column of $y$-values, the active field must be nonzero and the additional field must 0 . To write two sets of $y$-values, the additional field must also be non-zero. To write three sets of $y$-values, both the active and additional unit numbers must be 0 , in which case the current unit number will be used to create the first column, and the next two adjacent existing unit numbers will be used to create columns 2 and 3 in the .DAT file. A simple module UACTADD0 (Utility, set ACTive and ADDitional to 0 ) will reinitialize the unit numbers so that up to three columns of data might be written.

All research modules create a.OUT file, with filenames identical to the module names, that contain information about the execution flow of the module. Parameters used and the unit numbers accessed or created are listed in these files, so that an orderly cross-referencing can be conducted if some of the results are brought into question. In addition, these modules record their activity in a history file (.HST) so that the sequence of executions can be checked at a later time.

\section{Research Modules}

In Table 2 we list the currently existing modules. These modules were designed 
in the course of a research project where the goal was to understand the propagation of errors in near-field data to the far field, and to develop techniques to remove the effects of these errors from the far field. Thus, some of these modules are very specific to this research projects; others, however, have more general applicability.

To illustrate the use of these modules in research, we provide first a simple, then a more elaborate example of computational sequence that delivers results required by two representative research problems.

\section{Simple research problem.}

Given a near field dataset, obtain a perspective plot of both the near field and the far field of the antenna.

Using ' $x$ ' to mean 'execute' a module, this simple task would be accomplished by entering the following batch commands at the DOS prompt:
$\mathrm{x}$ uinitun
$x$ urdnfff
$\mathrm{x}$ ucbdgrd
plt ff
$\mathrm{x}$ uswtonf
$\mathrm{x}$ ucbdgrd
plt nf

Here $p l t$ is a DOS batch file that calls on the plot package on the system to process the graph data files output by UCBDGRD. Thus, this part of the procedure would vary from system to system, depending on the graphics package used.

From Table 2 we can easily ascertain that the above sequence of computational steps above will deliver the results required. First, by executing UINITUN we initialize the system variables and unit numbers to delete the results of all previous executions of modules. Next, we read in the original near-field dataset and transform it to the far field. At this point, the data management system sets the ffornf variable to $f f$, since the last field created was a far field. Now UCBDGRD will access the far-field dataset to create a plot file. To create a plot file using the current near field, we must set the system variable ffornf to $n f$. Hence, we execute USWTONF, and then UCBDGRD will access the near-field dataset to create a plot file for the near field.

A more complicated research problem.

Given a near-field dataset, introduce known errors into this near field. Use a known probe position error function and the Taylor series technique to generate errorcontaminated near-field values. Then, remove these errors from the data using a well defined error correction technique, and compare the error-free, error-contaminated and error-corrected near and far fields by looking at the respective complex ratios of field values at each data point. Present the results in perspective plots and/or linear plots showing ratios of amplitudes and phase differences. 
Using the existing set of research modules, this relatively involved research task can be brought to conclusion as follows:

x uinitun
x umakedz
x urdnfff
x uts
x ec

Executing this sequence, we have accomplished the first part of the research. Again, we started by initializing the system parameters and unit numbers. Then, a probe displacement error field has been created by executing UMAKEDZ. The function to be used is defined in the parameter file read by the module. This is listed in Table 4. A . GRD file to draw a perspective plot of the error function has also been created. Next, the near-field dataset is read in and the corresponding far field is calculated. Then errors are introduced into the original near field using the Taylor series technique by executing the module UTS, and the errors are removed by inverting the error operator when the module EC is executed. At this point each dataset has been recorded on the disk in complex binary dataset files with filenames fort and file extensions . $x x$, where $x x$ is some unit number automatically assigned by the data management section of the system. We can now proceed to obtain the far field corresponding to each near field created up to now. We proceed as follows:

$$
\begin{aligned}
& x \text { udecnf } \\
& x \text { urdnfff } \\
& x \text { uincnf } \\
& \text { x urdnfff }
\end{aligned}
$$

All far fields of interest have now been created. By executing UDECNF, the current near field unit number has been decremented by 1 (assuming that the unit increment/decrement parameter is 1 , the default), thereby making the near field obtained prior to the last near field current. Then executing URDNFFF will transform this near field into a far field. Incrementing the near-field unit number will increase the current unit number by 1 , which, in this case, is the last near field created. Again executing URDNFFF will create the corresponding far field.

Only plotting and comparing the various near fields and far fields is left. The module UDIVCBD can be used to form the complex ratio of two near-field or farfield datasets. As discussed above in the data management section, the two desired datasets are loaded by defining an active and additional unit numbers, or if these are set to 0 , then the two most recently created fields (near or far) will be used. Thus, to take the ratio of the error-contaminated near field to the original near field, we execute the following:

$$
\begin{aligned}
& \mathrm{x} \text { uswtonf } \\
& \mathrm{x} \text { uactts } \\
& \mathrm{x} \text { uaddnfo } \\
& \mathrm{x} \text { udivcbd }
\end{aligned}
$$


Similarly, to take the ratio of the error-corrected near field and of the original near field we execute the following:

$\mathrm{x}$ uactec

$\mathrm{x}$ uaddnf0

$\mathrm{x}$ udivcbd

In both of the above sequences of operations the complex ratio field is created, which is recorded sequentially using near-field unit numbers, since we executed USWTONF at the beginning of this sequence. Note that the second execution of UADDNF0 is really redundant, since it was already executed above.

To create far-fields ratios the procedure is somewhat different, since far fields have not been labeled by special identifiers, such as ts and ec. Any far field can be made current by incrementing or decrementing the far-field unit numbers an appropriate number of times, and can be selected by executing one of the modules UACTFF or UADDFF. Thus, to form all ratios we execute the following sequence:

$x$ uswtoff
$x$ uaddff 0
$x$ uincff
$x$ uactff
$x$ udecff
$x$ udivcbd
$x$ uincff
$x$ uactff
$x$ udecff
$x$ udivcbd

All far-field ratios of interest have now been created and recorded on farfield unit numbers. This was accomplished by first switching to the far fields (USWTOFF), then making the original far field the additional field (UADDFF0), followed by making the far field created before the last one the active field (UINCFF, $\mathrm{UACTFF}$ and UDECFF) and taking the ratio (UDIVCBD). After the ratio was taken the current far field unit number was automatically increased. Next, the previously created far field was made current (UDECFF) and active (UACTFF), the current unit number reincremented (UINCFF) and then the ratio (UDIVCBD) was taken. Each ratio field was automatically recorded on next available far-field unit number.

At this point we can obtain a system status report, so that any problem with the sequence of operations could be detected. For this purpose we execute the module USHOWUN, whose output is presented in the second table in Appendix C, with a detailed discussion.

After examining the output of USHOWUN and ascertaining that no errors were made, we can proceed to plot any of the existing fields (fort.xx files). First, an ascii plot file (.GRD) needs to be created using the module UCBDGRD, after which plots can be created using the plot package. The module UCBDGRD will 
read the current far or near field depending on the setting of the switch ffornf. A setting can be selected by executing USWTOFF or USWTONF. Alternatively, a unit number can be loaded by making it active by executing one of the number of modules which have the phrase ACT embedded in their names.

Sample plotting procedures would be as follows:

x uswtonf

$\mathrm{x}$ unorm 1

$x$ ucbdgrd

plt nf

or

$\mathrm{x}$ uswtonf

$\mathrm{x}$ unorm0

$\mathrm{x}$ uactts

$x$ ucbdgrd

plt nf

or

$\mathrm{x}$ uswtoff

$\mathrm{x}$ uact0

$x$ ucbdgrd

plt ff

In all these examples we first specify the type of fields we want to access. Then, in the first example, we set the normalization constant to 1 , since we are plotting a ratio field, which does not have to be normalized when it is converted to decibels. Next, a plot file is created by UCBDGRD. In the second example, the normalization constants are restored to their proper values (UNORM0), then the error-contaminated near field created by the Taylor series method is activated (UACTTS), and a plot file is created. In the third example, we switch to the far field, zero out the active unit number so that the current far field is accessed by UCBDGRD to create the plot file. In all three cases, we use the DOS batch command $p l t$ to plot either the far field ( $f f)$ or the near field $(n f)$.

5. Output Files.

All research modules have been constructed to write an output file where the parameters and data files used during execution are clearly listed. This way the settings of input/output parameters can be cross-referenced, and the correctness of the computational sequence and numerical inputs can be ascertained. These output files have the name of the modules as their filenames and .OUT for the file extension.

Certain modules write ascii datasets to be used by the graphics package on the system. The module UCBDGRD creates two-dimensional ascii datasets for perspective and contour plots, and the module UCBDDAT creates ascii datasets (.DAT) for simple $x y$-plots. The module URMSCBD creates a .DAT file to plot the rms distribution of the power radiated in a far field. These .GRD and .DAT ascii 
files may also be used to examine the data for any features we might be interested in.

Finally, the module UPRNCBD prints the rows and/or columns of any far- or near-field CBD file, according the switch setting of ffornf and the settings of the current and active unit numbers. If the active unit number is 0 , then the current file will be printed. The particular rows and/or columns to be printed and the respective ranges of data are set by the parameter file sub.prn.

\section{DOS Batch Files}

DOS batch files can be used to advantage to save time and effort when performing step-by-step computations to obtain a result. We can write batch files merely as abbreviations of longer commands, or to collect a set of executable steps that will be used many times over. The complexity of the batch files and their usefulness are limited only by the programmer's knowledge of the DOS operating system and the programmer's imagination.

The use of the plt.bat file has been illustrated in the previous section a number of times. Another example of a batch file is the abbreviation of the execution of the first simple research problem discussed above. Thus, the batch file pltnfff would look like this:

command / $\mathrm{c}$ u uinitun command /c x urdnfff command / $\mathrm{c}$ x ucbdgrd command /c x uswtonf command / $\mathrm{c}$ x ucbdgrd command /c plt nf command /c plt ff

Simply typing pltnfff at the DOS prompt would execute all the steps in this batch file. We now have a very easily usable, high level program that will produce plots of the near and far fields of the current dataset. The DOS expression command $/ c$ is used here to continue execution within the batch file to the last line. Without command / $c$ execution would not return to the next step, but exit to the DOS prompt.

The second research problem is the implementation of the error-correction problem using a specific error-creation and error-correction technique. What might change from one implementation to the next is the original dataset to be used, the form of the error function and the magnitude of the error function. These are all inputs to the complete procedure; that is, the program execution steps are the same, independent of these parameters. Therefore, a DOS batch file is appropriate for recording the steps of this relatively complicated research project. This batch file could be appropriately called error.bat (error correction), and would look like this:

command / $\mathrm{x}$ uinitun

command /c x umakedz 


command $/ \mathrm{c} \times$ urdnfff
command $/ \mathrm{c} \times$ uts
command $/ \mathrm{c} \times \mathrm{x}$ ec
command $/ \mathrm{c} \times$ udecnf
command $/ \mathrm{c} \times$ urdnfff
command $/ \mathrm{c} \times$ uincnf
command $/ \mathrm{c} \times$ urdnfff
command $/ \mathrm{c} \times$ uswtonf
command $/ \mathrm{c} \times$ uactts
command $/ \mathrm{c} \times$ uaddnf0
command $/ \mathrm{c} \times$ udivcbd
command $/ \mathrm{c} \times$ uactec
command $/ \mathrm{c} \times$ udivcbd
command $/ \mathrm{c} \times$ uswtoff
command $/ \mathrm{c} \times$ uaddff
command $/ \mathrm{c} \times$ uincff
command $/ \mathrm{c} \times$ uactff
command $/ \mathrm{c} \times$ udecff
command $/ \mathrm{c} \times$ udivcbd
command $/ \mathrm{c} \times$ uincff
command $/ \mathrm{c} \times$ uactff
command $/ \mathrm{c} \times$ udecff
command $/ \mathrm{c} \times$ udivcbd

This batch file goes as far as creating all the required near and far fields of the research project, as well as the ratio fields. It stops short of plotting any of the existing fields. A separate batch file would be appropriate for creating a desired set of plots.

The batch files using the executable modules of the PNFC allows one to create and save complicated research procedures in a straightforward and efficient manner. A collection of such batch files over a period of time would greatly enhance the computational ability and efficiency of any research group.

7. Symbol Definitions

To help the user understand the names of the modules and subroutines used in the PNFC, we compiled a table of symbols with their most commonly used definitions. This list is presented in Table 6 . This table should make reading the source codes easier. We hope that authors of new code will use existing symbols as far as possible to contribute to the coherence of the full package.

\section{Subroutine Descriptors}

In Table 7 we compiled a list of subroutines along with brief descriptors of their functions. This can be helpful when creating new modules or when planning to write new subroutines to perform computational tasks not yet addressed in the package. 


\section{Table of Dependencies}

In Appendix $\mathrm{D}$ a table of dependencies showing the interrelationship between the various subroutines is presented. Primarily this table can serve as an index of subroutines file, since all existing subroutines are included alphabetically in the leftmost column. The subroutines called by the routine on the left are listed following the colon in the order in which they are called. In this manner we can get an overview of both the contents and structure of the-complete code. Such a file can be used to advantage when developing new code, or when improvements in the existing code are contemplated.

\section{Summary}

In this report we have outlined and documented the computational structrures and procedures of a newly created software package named Planar Near Field Codes (PNFC) for personal computers. This package supports the computational effort needed to solve research problems in antenna metrology.

The PNFC can be used to address diverse research problems because of its highly modular structure wherein independent subroutines have been combined to create independent research modules. These modules have been constructed to provide the computational procedure for recurring research themes in antenna metrology as well as for research problems that arose in connection with the specific task of correcting for probe position errors in planar near field data. A data management procedure has been implemented that automatically keeps track of the various datasets being created and stored during the course of research. Because of the highly modular nature of the PNFC new research modules can be easily constructed and incorporated into the total system. A large number of independent subroutines are available to support new efforts, and new subroutines can be added without any difficulty.

Streamlining computational procedures along the lines built into this software package can result in significant reduction of time needed to obtain answers to complicated research problems. Additions to the current version of the package over an extended period of time would create a truly comprehensive computer package capable of dealing with most computational needs of antenna metrology easily. For this reason all users are encouraged to add to the effort as they see appropriate. 
Table 1.

Definition of Symbols Used in Naming Modules

SYMBOL

0

1

2

act

act0

add

add0

amp

ap

cbd

cor

$\mathrm{db}$

dif

div

drv

ds

dacb

dat

$\mathrm{dbp}$

dc

dec

deriv

dif2

difa

$\mathrm{d} z$

ec

err

ff

ff0

grd

hst

inc

init

laplen

make

nf

nfo
MEANING

initial

set to 0

version 1

squared quantity

active, activate

set ACTive switch to 0

additional

set ADDitional switch to 0

amplitude

amplitude, phase

complex binary dataset

correct, corrected, correction

in $\mathrm{dBs}$

difference

divide, divided (ratio)

derivative

direct sum

direct access complex binary (file)

.DAT (file)

$\mathrm{dB}$, phase complex storage

decrement

decrement

derivative

difference between squared amplitudes

difference in amplitude

function $\mathrm{dz}$

error correction

error

far field

original far field

.GRD (DOS file extension)

history

increment

initialize

Laplacian

near field

original near field 
nc

norm0

norm1

op

prn

$\mathrm{rbd}$

rd

rms

show

sw

to

ts

$\mathrm{u}$

un increment

normalization of original datasets

normalization with 1

operator

print

real binary dataset

read

root mean square

switch

to

Taylor series

utility

unit number 
Table 2

List of Modules That Perform Basic Computational Tasks

UAMP2CBD read a near-field or a far-field dataset and write its squared amplitude to a complex binary data file

UAPDACB read an amplitude, phase ascii file and write a direct access complex binary file

UCBDDAT read a complex binary data file and create a .DAT file for $x-y$ plots

UCBDGRD

UDBPDACB read a complex binary data file and create a .GRD file for two dimensional contour or surface plotting

read a $d B$,phase ascii file and write a direct access complex binary file

UDERIV read a near-field dataset and write the derivative of some specified order

UDIF2CBD read two far-field or near-field datasets and write the difference of the squared amplitudes to a CBD file

UDIFACBD read two far-field or near-field datasets and write the difference of the amplitudes to a CBD file

UDIFCBD read two far-field or near-field datasets and write the complex difference to a CBD file

UDIFDB read two far-field or near-field datasets and write the difference of amplitudes in $\mathrm{dBs}$ and the phase difference to a CBD file

UDIVCBD read two far-field or near-field datasets and write the complex ratio to a CBD file

UDIVRBD read two real binary data files and write the ratio to a RBD file

UDS

UERR

UERRCOR2 create a near-field dataset with errors in it using the direct sum algorithm

create a near-field dataset with errors in it using the Taylor series method

ULAPLCN

UMAKEDZ

UOPNORM read a near-field dataset with errors in it and do a second order error correction

UPRNCBD

URBDGRD

URDDZ

URDFFNF

URDNFFF

read a near-field dataset and form the Laplacian and check that it satisfies the scalar wave equation

URMSCBD

create an array DZ using a specified error function and write a GRD file to plot the error function

calculate the norm of the error operator

print specified rows and columns of a complex binary data file read a real binary dataset and create a grid file for plotting read the error function file $\mathrm{dz}$ and create a GRD file for plotting read a far field and transform it to the near field read a near field and transform it to the far field sum the rms values at grid points of a CBD file, and create a .DAT file for plotting 
USUBGRD convert a specified part (SUB) of a CBD file to a GRD file for plotting

UTS introduce errors into a near-field dataset using the Taylor series method 
Table 3.

List of Modules That Perform Basic Data Management Functions.

UACT0

UACTADD0

UACTDDB

UACTDIF

UACTDIV

UACTDRV

UACTDS

UACTEC

$\mathrm{UACTFF}$

UACTFF0

UACTNF

UACTNF0

UACTTS

UADD0

UADDDRV

UADDDS

UADDEC

UADDFF

UADDFF0

UADDNF

UADDNF0

UADDTS

UDCDFDV

UDECFF

UDECNF

UINCFF

UINCNF

UINITUN

UNCDFDV

UNORMO

UNORM1

URESTFF

URESTNF

USAVEFF

USAVENF

USETDB1

USETDB2 set the active unit number to 0 set the active and additional unit numbers to 0 set the active unit number to the value in difdb.iun set the active unit number to the value in dif.iun set the active unit number to the value in div.iun set the active unit number to the value in $d r v$.iun set the active unit number to the value 0 set the active unit number to the value in ec.iun set the active unit number to the final value in ff.iun set the active unit number to the initial value in ff.iun set the active unit number to the final value in $n f$.iun set the active unit number to the initial value in $n f . i u n$ set the active unit number to the value in ts.iun set the additional unit number 0 set the additional unit number to the value in drv.iun set the additional unit number to 0 set the additional unit number to the value in ec.iun set the additional unit number to the final value in ff.iun set the additional unit number to the initial value in ff.iun set the additional unit number to the final value in $n f$.iun set the additional unit number to the initial value in $n f$.iun set the additional unit number to the value in ts.iun decrement the unit number recorded in difdiv.iun decrement the value of the current far-field unit number decrement the value of the current near-field unit number increment the unit number recorded in ff.iun increment the unit number recorded in nf.iun initialize the system parameters and unit numbers increment the unit number recorded in difdiv.iun set the far-field and near-field normalization constants to their initial values

set the far-field and near-field normalization constants to unity restore the unit numbers in ff.iun to the values saved in save.ffs restore the unit numbers in $n f$.iun to the values saved in save.nfs save the unit numbers recorded in ff.iun in save.ffs save the unit numbers recorded in nf.iun in save.nfs copy the first set of values of dbctoff, dbfloor in dbmins.set to $d b$ $\min . d b$

copy the second set of values of dbctoff,dbfloor in dbmins.set to dbmin.db 
USETDB3 copy the third set of values of dbctoff,dbfloor in dbmins.set to $d b$ $\min . d b$

USHOWUN display on the screen the current system parameter settings and the current unit settings

USWBOTH record the value ffnf into ffornf.iun

USWFFNF toggle the value recorded in ffornf.iun between $f f$ and $n f$

USWTOAMP record the value $a m p$ in ampordb.grd

USWTODB record the value $d B$ in ampordb.grd

USWTODS record the value $d s$ in dsorts.iun

USWTOFF record the value ff in ffornf.iun

USWTONF record the value $n f$ in ffornf.iun

USWTONON record the value none in ampordb.grd

USWTOTS record the value $t s$ in dsorts.iun 
List of Parameter Files Used by the Research Modules and the Data Files They Create

module

parameter files

$\begin{array}{ll}\text { UAMP2CBD } & \text { dabd.iof } \\ \text { UAPDACB } & \text { adab.iof } \\ \text { UCBDDAT } & \text { dabd.iof, ampordb.grd } \\ & \text { ampnorn.nf, ampnorm.ff } \\ & \text { difdiv.iun, dbmin.db } \\ & \text { dbloss.grd, iregion.nf } \\ & \text { iregion.ff }\end{array}$

UCBDGRD ampordb.grd, ampnorm.nf

ampnorm.ff, dbloss.grd

dbmin.db , dabd.iof

UDBPDACB adab.iof

UDERIV order.drv, dabd.iof, sub.grd

dbmin.db

UDIF2CBD difdiv.iun, dabd.iof

UDIFACBD difdiv.iun, dabd.iof

UDIFCBD difdiv.iun, dabd.iof

UDIFDB difdiv.iun, dabd.iof, ampnorm.ff ampnorm.nf, dbmin.db

UDIVCBD difdiv.iun, dabd.iof, iregion.ff iregion.nf

UDIVRBD difdiv.iun, dabd.iof, iregion.ff iregion.nf

UDS

UERR

UERRCOR

ULAPLCN

UMAKEDZ dabd.iof, fun.dz, polydz.par

UOPNORM difdiv.iun, dabd.iof, iregion.ff

UPRNCBD dabd.iof, sub.prn

URBDGRD dabd.iof

URDDZ

URDFFNF perdz.par, randz.par iregion.nf

sub.ds, filter.ff, scale.dz, dabd.iof

dabd.iof, scale.dz

dabd.iof, scale.dz

dabd.iof, dbmin.db

dabd.iof, makedz.par, scale.dz

filter.ff, dabd.iof, db.ff, db.nf data files

uamp2cbd.out, fort. $x x^{1}$

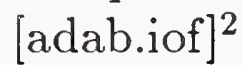

nfyamp.dat, nfyphase.dat nfxamp.dat, nfxphase.dat ffyamp.dat, ffyphase.dat ffxamp.dat, ffxphase.dat ucbddat.out nfamp.grd, nfphase.grd ffamp.grd, ffphase.grd ucbdgrd.out [adab.iof] ${ }^{2}$ order.drv, uderiv.out, fort. $x x^{1}$ drvamp.grd, drvphase.grd udif2cbd.out, fort. $x x^{1}$ udifacbd.out, fort. $x x^{1}$ udifcbd.out, ' fort. $x x^{1}$ udifdb.out, ddbamp.grd, fort. $x x^{1}$ ddbphase.grd udivcbd.out, fort. $x x^{1}$

udivrbd.out, fort. $x x^{1}$

scale.dz, uds.out, fort.ud $x^{1}$ uerr.out, scale.dz, fort. $x x^{1}$ scale.dz, uerrcor.out, fort. $x x^{1}$ ulaplcn.out, lnamp.grd, lnphase.grd amp0.grd, phase0.grd iregion.ff, iregion.nf, umakedz.out $[\mathrm{dz} . \mathrm{grd}]^{2}$, fort. $x x^{1}$ uopnorm.out

uprncbd.out

ffamp.grd, nfamp.grd, urbdgrd.out scale.dz, urdz.out, [dz.grd $]^{2}$ iregion.ff, iregion.nf, fort. $x x^{1}$ urdffnf.out, db.ff, fort. $x x^{1}$ 
URDNFFF filter.ff, dabd.iof, db.ff, db.nf

URMSCBD dabd.iof, ampnorm.ff

USUBGRD dbloss.grd, dabd.iof, sub.grd ampnorm.nf, ampordb.grd ampnorm.ff, dbmin.db

UTS ampnorm.ff, db.nf, ampnorm.nf iregion.ff, iregion.nf, fort. $x x^{1}$ urdnfff.out, db.ff, fort. $x x^{1}$ ampnorm.ff, db.nf, ampnorm.nf db.ff, db.nf, urmscbd.out, fort. $x x^{1}$ ampnorm.ff, rms.dat

ffamp.grd, ffphase.grd, nfamp.grd nfphase.grd, usubgrd.out

uts.out, scale.dz, fort. $x x^{1}$

1 The DOS extension number $x x$ added to the filename FORT is recorded in the appropriate .IUN file

2 The brackets [filename] is to be understood as the contents of the filename. For example, the output file name is read in as a parameter from file adab.iof 
Table 5

List of Parameter Files Used by the Data Management Modules

module input file

UACT0

UACTADD0

UACTDDB

UACTDIF

UACTDIV

UACTDRV

UACTDS

UACTEC

UACTFF

UACTFF0

UACTNF

UACTNF0

UACTTS

UADD0

UADDDRV

UADDDS

UADDEC

UADDFF

UADDFF0

UADDNF

UADDNF0

UADDTS

UDCDFDV

UDECFF

UDECNF

UINCFF

UINCNF

UINITUN

UNCDFDV

UNORM0 difdb.iun

dif.iun

div.iun

drv.iun

- ERROR ${ }^{1}$ -

ec.iun

ff.iun

ff.iun

nf.iun

nf.iun

ts.iun

drv.iun

- ERROR ${ }^{1}$ -

ec.iun

ff.iun

ff.iun

nf.iun

nf.iun

ts.iun

difdiv.iun, ffornf.iun

ff.iun, nf.iun

ff.iun

nf.iun

ff.iun

nf.iun

init.iun, data.dir output file

active.iun

active.iun, add.iun

active.iun

active.iun

active.iun

active.iun

active.iun

active.iun

active.iun

active.iun

active.iun

active.iun

active.iun

add.iun

add.iun

add.iun

add.iun

add.iun

add.iun

add.iun

add.iun

add.iun

difdiv.iun

ff.iun

nf.iun

ff.iun

nf.iun

ampordb.grd, filter.ff, order.drv

scale.dz, fun.dz, active.iun, add.iun amp2.iun, asci.iun, difdiv.iun dif2.iun, dif.iun, difdb.iun, div.iun drv.iun, ds.iun, dz.iun, ec.iun, err.iun ff.iun, nf.iun, rdiv.iun, ts.iun difdiv.iun

tempnorm.nf, ampnorm.nf tempnorm.ff, ampnorm.ff 


$\begin{array}{lll}\text { UNORM1 } & \text { ampnorm.nf, ampnorm.ff } & \text { tempnorm.nf, ampnorm.nf } \\ & \text { tempnorm.nf, tempnorm.ff } & \text { tempnorm.ff, ampnorm.ff } \\ \text { URESTFF } & \text { save.ffs } & \text { ff.iun } \\ \text { URESTNF } & \text { save.nfs } & \text { nf.iun } \\ \text { USAVEFF } & \text { ff.iun } & \text { save.ffs } \\ \text { USAVENF } & \text { nf.iun } & \text { save.nfs } \\ \text { USETDB1 } & \text { dbmins.set } & \text { dbmin.db } \\ \text { USETDB2 } & \text { dbmins.set } & \text { dbmin.db } \\ \text { USETDB3 } & \text { dbmins.set } & \\ \text { USHOWUN } & \text { ampordb.grd, filter.ff, fun.dz } & \\ & \text { ampnorm.ff, ampnorm.nf } & \\ & \text { ffornf.iun, active.iun } & \\ & \text { difdiv.iun, dif2.iun, dif.iun } & \\ & \text { div.iun, drv.iun, ds.iun } & \\ & \text { err.iun, ff.iun, nf.iun, ts.iun } & \\ & \text { dz.iun, order.drv, amp2.iun } & \\ & \text { rdiv.iun, difdb.iun, asci.iun } & \\ \text { USWBOTH } & \text { scale.dz, add.iun, ec.iun } & \text { ffornf.iun } \\ \text { USWFFNF } & \text { ffornf.iun } & \text { ffornf.iun } \\ \text { USWTOAMP } & & \text { ampordb.grd } \\ \text { USWTODB } & & \text { ampordb.grd } \\ \text { USWTODS } & & \text { dsorts.iun } \\ \text { USWTOFF } & & \text { ffornf.iun } \\ \text { USWTONF } & & \text { ffornf.iun } \\ \text { USWTONON } & & \text { ampordb.grd } \\ \text { USWTOTS } & & \text { dsorts.iun } \\ & & \end{array}$

1 Output from module UDS is written to file FORT.DS $x$, where $x$ denotes a single digit. Consequently these unit numbers do not fit into a purely integer unit numbering scheme. 
Table 6

\section{List of Symbols Used by PNFC Subroutines}

$\mathrm{cm}$

cr

da

$\mathrm{db}$

df

ds

$\mathrm{dx}$ initialization designator

one dimensional, subsequent operation designator, alternate procedure designator two dimensional

"a", access, array, ascii, amplitude "b", backward, binary

change, convert to, column, complex, constant, copy data, derivative, difference, dimensional, direct (access), disk (as a storage location), double precision exponential, even far, field, file, forward, formated, function gamma, generate imaginary, imaginary part, integer $\mathrm{k}$ (integer constant), wave number, spectrum space $\mathrm{k}$ 1 (integer constant)

$\mathrm{m}$ (integer constant), maximum, minimum, minus, multiple near (fresnel region), negative quantity odd, or parameter(s), phase, plot, plus, power, print, product, pseudo read, real (single precision), real part, row shift, shifted, single precision, store, sum, plural designation taylor (series), times, transform

weight, weighted, write coordinate (distance along $\mathrm{x}$ axis), general variable designation name change letter (to avoid conflicts) coordinate (distance along y axis) coordinate (distance along $\mathrm{z}$ axis) ascii

binary data complex array "a" complex array "b" complex constant character variable centimeter create direct access decibel difference direct sum derivative with respect to $\mathrm{x}$, increment in $\mathrm{x}$ direction 


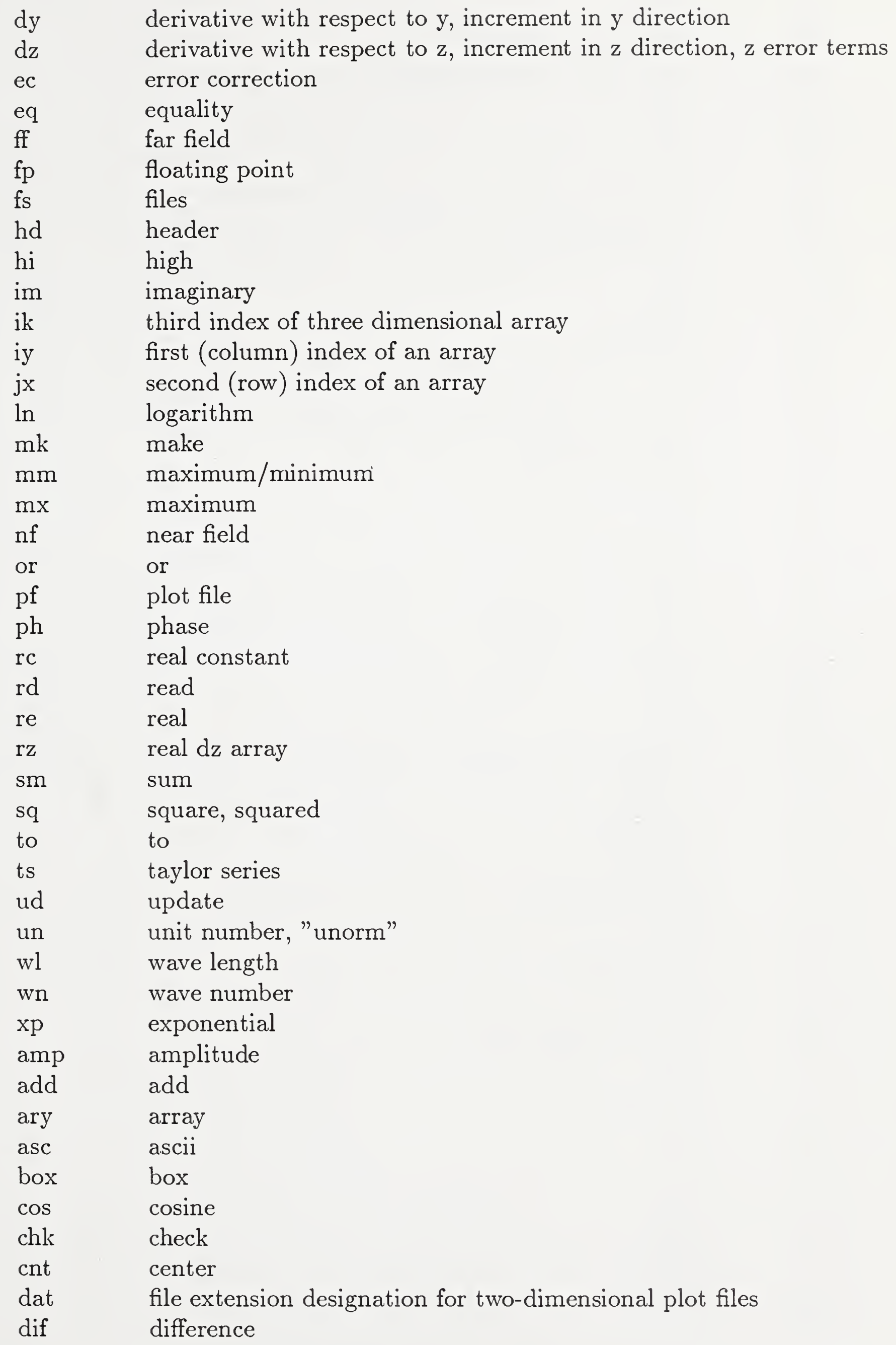




$\begin{array}{ll}\text { div } & \text { divide } \\ \text { dnf } & \text { derivative of near field } \\ \text { dot } & \text { dot product (of two vectors) } \\ \text { drv } & \text { derivative } \\ \text { end } & \text { end } \\ \text { err } & \text { error, error field } \\ \text { exp } & \text { exponent } \\ \text { fbt } & \text { forward/backward transform } \\ \text { fft } & \text { fast fourier transform } \\ \text { fil } & \text { file } \\ \text { flt } & \text { filter } \\ \text { fun } & \text { function } \\ \text { get } & \text { get } \\ \text { grd } & \text { file extension designation for perspective-plot files } \\ \text { hst } & \text { history } \\ \text { iof } & \text { input/output file } \\ \text { img } & \text { imaginary } \\ \text { inp } & \text { input } \\ \text { ins } & \text { insertion } \\ \text { int } & \text { integrate } \\ \text { iun } & \text { integer unit number } \\ \text { leq } & \text { less than or equal } \\ \text { log } & \text { logarithm } \\ \text { mak } & \text { make } \\ \text { mul } & \text { multiply } \\ \text { mod } & \text { modulate, modulated by } \\ \text { out } & \text { output } \\ \text { par } & \text { parameter } \\ \text { per } & \text { periodic } \\ \text { pff } & \text { psuedo far field } \\ \text { plt } & \text { plot } \\ \text { ply } & \text { polynomial } \\ \text { prg } & \text { program } \\ \text { prn } & \text { print } \\ \text { pws } & \text { plane-wave spectrum } \\ \text { scl } & \text { scale } \\ \text { set } & \text { set, setup } \\ \text { sft } & \text { shift } \\ \text { sin } & \text { sine } \\ \text { str } & \text { store } \\ \text { aray } & \text { array } \\ \text { bndr } & \text { boundry } \\ \text { char } & \text { character } \\ \text { gama } & \text { gamma } \\ & \end{array}$




$\begin{array}{ll}\text { gamm } & \text { gamma } \\ \text { file } & \text { file } \\ \text { fils } & \text { files } \\ \text { find } & \text { find } \\ \text { fltr } & \text { filter } \\ \text { func } & \text { function } \\ \text { grid } & \text { grid (coordinate grid) } \\ \text { init } & \text { initalize } \\ \text { limt } & \text { limit } \\ \text { loss } & \text { loss } \\ \text { make } & \text { make } \\ \text { mult } & \text { multiply } \\ \text { mess } & \text { message } \\ \text { prnt } & \text { print } \\ \text { rndm } & \text { random } \\ \text { swap } & \text { swap } \\ \text { unit } & \text { unit } \\ \text { gamma } & \text { gamma } \\ \text { polyn } & \text { polynomial } \\ \text { ratio } & \text { ratio } \\ \text { const } & \text { constant } \\ \text { lngth } & \text { length } \\ \text { range } & \text { range } \\ \text { timer } & \\ \text { gather } & \\ \text { laplcan } & \text { Laplacian } \\ \end{array}$




\section{TABLE 7}

\section{List of Subroutines of the PNFC}

ACPCFFD

ACPCNFD

ADABIOF

ADABPAR

ADDBOX

AMPDIF2

APDSET1

CABD

CABDIOF

CABDPAR

CADACB

CADD1

CADD2

CAEIPH2

CAEIPHC

CAPCCD1

CAPRI

CAPRI1

CAPRNT

CARAYMX2

CARCBD2

CATOCB2

CBDTODB

CCA2B1

CDFSET1

CDIF1

CDIF2

CDIVDS2

CDOT

CGATHER

CHKEQFP

CHKEQI

CHKLEQI

CHKPAR0

CHKPAR1

CHKPAR2 introduce Amplitude Change and Phase Change to Far-Field Data introduce Amplitute Change and Phase Change to Near-Field Data get the Input Output File for Ascii to Direct Access Binary routines read the PARameters for the Ascii to Direct Access Binary conversion ADD a BOX function of amplitude EPS to the complex CDATA form a real array equal to the difference between the absolute values of two complex arrays

convert to A-P prior to taking Difference between SElected columns or rows from Two 2-dimensional arrays created from 3-dimensional array Convert two Ascii data sets to self-documented complex Binary Data set get the Input Output File for the Conversion of Ascii to Binary Data read PARameters for the Conversion of Ascii to Binary Data routines Convert 2 Ascii data sets to self-document Direct-Access Complex Binary Complex ADDition of 1 dimensional complex arrays Complex ADDition of 2 dimensional complex arrays Complex Array times E to power I times Phase, phase 2 dim. real array Complex Array times Exponential I times Phase Constant Complex Array Plus Complex Constant, 1 Dimensional Convert Amplitude-Phase format complex numbers to Real-Imaginary format

Convert A-P format complex numbers to R-I format, 1 dimensional find max and min values of a Complex Amplitude-Phase array and $\mathrm{pRiNT}$ find the Complex ARrAY MaXimum, for a 2 dimensional array Complex Array plus Real weight times Complex array B, dbl. precision copy a Complex array CA TO CB, for 2 dimensional arrays read a Complex Binary data set, converting from $\mathrm{R}-\mathrm{I}$ TO A-P, amp in $\mathrm{dB}$ copy a Complex Column of data from array A 2 dim. to array B $1 \mathrm{dim}$. form Complex DiFerence of SElected columns or rows of Two arrays formed from a 3-dimensional's sub-arrays, then convert from R-I to A-P Complex DIFerence of 1 dimensional complex arrays Complex DIFerence of 2 dimensional complex arrays Complex DIVision of single precision array by Double precision array Complex Dot product returned in complex ANSwer sequentially copy regularly spaced elements of one array to another CHecK for EQuality between two Floating Point numbers: stop if unequal $\mathrm{CHecK}$ for EQuality between two Integer numbers: stop if unequal CHecK if Less than or EQual relates two Integer numbers: stop otherwise $\mathrm{CHecK}$ if one integer is less than or equal to another: stop otherwise CHecK if two integers are less than or equal to two others: else stop CHecK if two integers are less than or equal to two others: else stop 
CHLNGTH

CIMGSTR

CINIT1

CINIT2

CIRCFLT

CMULDS2

CMULRD2

CMULT1

CMULT2

CMULTR2

CNIMCC1

CNTCACB

CONST

CONSTAX

COS2

COS3

COS4

COSAX

COSAX2

COSX

CRA2B1

CRATIO2

CRIAP

CRIAP1

CRNFERR

CSMWCP1

CSMWCP2

CSUM1

CSUM2

CSUMCP1

CSUMCP2

CSUMIK2

CSUMRW1

CSUMRW2

CSWCPE2

CXPCLOG

DABDIOF

DABDPAR

DATORB1

DB1

DB2

DCLN2

DNFDX determine number of CHaracters up to a blank in a character variable a Complex array's IMaGinary part is SToRed in a real array

Complex INITialization of 1 dimenstional array with a complex constant Complex INITialization of 2 dimenstional array with a complex constant CIRCular FiLTer of CDATA

Complex MULtiplication of a Double precision complex array by a Single precision complex array in 2 dimensions

Complex MULtiplication of Real Double precision array by complex array

Complex MULTiplication of complex arrays in 1 dimension

Complex MULTiplication of complex arrays in 2 dimensions

Complex MULTiplication of Real array by complex array in 2 dimensions

add a Complex Constant to Negative Imaginary part of Complex array

CeNTer the data in a zero padded array by copying it from $\mathrm{CA}$ to $\mathrm{CB}$

return the constant unity for the value of a function

return the constant unity for the value of a function

calculate the function COSine squared of $x$

calculate the function COSine cubed of $x$

calculate the function COSine of $\mathrm{x}$ raised to the fourth power

calculate the function $\operatorname{COS}\left(\mathrm{A}^{*} \mathrm{X}\right)$

calculate the function COSine squared of $\mathrm{A} * \mathrm{X}$

calculate the function $\operatorname{COS}(\mathrm{X})$

copy a Complex Row of data from array A $2 \mathrm{dim}$. to array B $1 \mathrm{dim}$.

Complex RATIO of two arrays in 2 dimensions

Complex Real-Imaginary format numbers to Amplitude-Phase format, $2 \mathrm{dim}$.

Complex Real-Imaginary format numbers to Amplitude-Phase format, $1 \mathrm{dim}$.

CReate ERRor Near Field via multiple Fourier transforms for all z dist.

Complex SuM of Weighted Complex Product real and complex arrays, $1 \mathrm{dim}$.

Complex SuM of Weighted Complex Product real and complex arrays, $2 \mathrm{dim}$.

Complex SUM in 1 dimension

Complex SUM in 2 dimensions

Complex SUM of Complex and real array Products, 1 dimensional result

Complex SUM of Complex and real array Products, 2 dimensional result

Complex SUM of complex array over 3rd dimension, 2 dimensional result

Complex SUM of a Real Weight times a complex array in 1 dimension.

Complex SUM of a Real Weight times a complex array in 2 dimensions

Weighted Complex Sum of ComPlex times real to Exponent in 2 dimensions add a Complex EXPonent array to the Complex LOGarithm of array CDATA get the Direct Access Binary Data Input Output File

obtain Direct Access Binary Data processing PARameters

copy a Double precision Array TO a Real array in 1 dimension

convert real part of amplitude-phase-format complex array to $\mathrm{dB}, 1 \mathrm{dim}$.

convert real part of amplitude-phase-format complex array to $\mathrm{dB}, 2 \mathrm{dim}$.

Double-precision Complex Logarithm of complex array in 2 dimensions

Derivative of Near Field with respect to X, a scan plane coordinate 
DNFDY

DNFDZ

DNFDZE

DNFDZO

DSPWS

ECEXP

EIGAMAZ

ERRMESS

FAXSBYS

FBTIOF

FBTPAR

FFNF

FFNFZXY

FFPFF

FFTFFT

FILSIOF

FILSPAR

FINDEND

FLTEIGZ

FLTGAMA

FLTLIMT

FLTPWSG

FLTRHIK

FRBW

FRGRD

FRRAD

FRRADHD

FUNAXBY

FUNCSCL

FUNCXY

FWDCRA1

FWRAD1

FWRAD2

GAMMASQ

GETFILE

GETWN

GRDDACB

GRID
Derivative of Near Field with respect to $\mathrm{Y}$, a scan plane coordinate multiple Derivatives of Near Field with respect to Z, coordinate variable orthogonal to the scan plane

Derivatives of Near Field with respect to Z, Even orders

Derivatives of Near Field with respect to Z, Odd orders

Direct Sum of Plane Wave Spectrum with log of spectrum as input data compute $\mathrm{E}$ to a Complex EXPonential

create the array $\mathrm{E}$ raised to the power $\mathrm{i}$ times GAMma times $\mathrm{Z}$

print a set of ERRor MESSages in accord with the origin of call

generate an array equal to a Function of A times Shifted coordinate X mul-

tiplied by a function of $B$ times Shifted coordinate $Y$

get Input Output File for real to complex binary data read routines

read PARameters for real to complex binary data read routines

given a Far-Field, compute the corresponding Near-Field using the FFT

given Far-Field, compute variable z-distance Near-Field via direct sum

given a Far-Field, obtain the Pseudo Far-Field

Fast Fourier Transform followed by inverse Fast Fourier Transform

get the Input Output File for reading designated data output file names

read the names of designated data-output files

skip to the end of a file

filter an array according to criteria regarding array GAMMA2

set array GAMMA2 to zero whenever its value is less than some constant obtain data-point-spacing criteria for limiting plane-wave spectrum

FiLTer sum of logarithm of Plane-Wave Spectrum plus i Gamma z

FiLTeR HIgh far-field frequencies in $\mathrm{K}$-space in near-field calculation; change from near-field plane at $\mathrm{Z} 0$ to near-field plane at Z1

perform a Formated Read and a Binary Write of a Real DATAset

perform a Formated Read of a .GRD DATAset with conversion from decibels to amplitude

perform a FoRmated Read of a Real Ascii Dataset

perform a FoRmated Read of a Real Ascii Dataset with HeaDer information generate an array equal to a FUNction of $\mathrm{A}$ times coordinate variable $\mathrm{X}$ multiplied by a function of $\mathrm{B}$ times coordinate variable $\mathrm{Y}$

calculate a SCaLed grid-increment

generate an array equal to a FUNCtion in $\mathrm{X}$ times a function in $\mathrm{Y}$

Formated Write of Double precision array Converted to Real Array, 1 dim.

Formated Write of Real Ascii Data in 1 dimension, self documented

Formated Write of Real Ascii Data in 2 dimensions, self documented

calculate real double precision array GAMMA**2

obtain the next file name from a file-name array

given a frequency, calculate a wavenumber

read amp and phase, GRD files and write out a Direct-Access Complex Binary Data file

set up a GRID using single precision along a single axis 
GRIDD

HSTDFDB

HSTDIF

HSTDIV

HSTDRV

HSTDS

HSTEC

HSTFFNF

HSTMKDZ

HSTNFFF

HSTTS

HSTUNO

HSTUN1

INPDABP

INPDACB

INPDRVP

INPDZP

INPFBT

INPFFP

INPFFP0

INPFILS

INPGRDP

INPNFP

INPNFP0

INPRCBD

INPTSP

INSLOSS

INTNF3

IUNIT

IYJXCNT

LAPLCAN

LNPPWS

MAKEDZ

MAKPFF

MDARB1

MDNFDX

MDNFDY

MDNFDZ

MDNFDZE

MDNFDZO

MIGAMMZ

MKGAMMA

MKPERDZ set up a GRID using Double precision along a single axis append file information to HiSTory file from program UDIFDB append file information to HiSTory file from program UDIFCBD append file information to HiSTory file from program UDIVCBD append file information to HiSTory file from program UDERIV append file information to HiSTory file from program UDS append file information to HiSTory file from program UERRCOR append file information to HiSTory file from program URDFFNF append file information to HiSTory file from program UMAKEDZ append file information to HiSTory file from program URDNFFF append file information to HiSTory file from program UTS append file information to HiSTory file from program UNORM0 append file information to HiSTory file from program UNORM1 INPut Direct-Access Binary data-processing Parameters from a file INPut Direct-Access Complex Binary data and parameters from a file INPut a subset of the direct-access pinary file for program UDERIV INPut a subset of the direct-access binary file for program UMAKEDZ INPut complex binary data by reading two real ascii files INPut Far-Field Parameter subset of direct-access binary data file INPut Far-Field Parameters and data from direct-access binary file get the file containing a list of file names and INPut the FILe nameS INPut a subset of the direct-access binary file for program UCBDGRD INPut Near-Field Parameter subset of direct-access binary data file INPut Near-Field Parameters and data from direct-access binary file INPut two Real data sets into a Complex Binary Data array INPut a subset of the direct-access binary file for program UTS convert INSertion LOSS from decibels to amplitude and scale data array INTegral of Near Field with respect to Z function call to increment by 1 the current Integer UNIT number determine the distance between the CeNTers of a line and a line segment along $\mathrm{X}$ or $\mathrm{Y}$ axes

calculate the LAPLaCiAN of a near field; store in adjacent location add i gamma times $z$ to Logarithm of Plane-Wave Spectrum and filter MAKE a function DZ, which is a function of $X$ and $Y$ MAKe a Pseudo Far Field equal to the Fourier transform of box function copy two Double precision Arrays to Real arrays in 1 dimension Multiple Derivatives of the Near Field with respect to X Multiple Derivatives of the Near Field with respect to $Y$ Multiple Derivatives of the Near Field with respect to $Z$ Multiple derivatives of the Near Field with respect to Z, Even orders Multiple derivatives of the Near Field with respect to Z, Odd orders calculate the array Minus i times GAMMa times $\mathrm{Z}$ $\mathrm{MaKe}$ (create) the arrays GAMMA**2 and $\mathrm{KY}, \mathrm{KX}$ MaKe a function DZ, which is a PERmutated function of $X$ and $Y$ 
MKPLYDZ

MMICA

MMRCA

NF

NFFF

NFMODX

NFMODY

NFPFF

OUTASC

OUTDACB

OUTDPS

OUTGRD

OUTPFS0

OUTPFS1

OUTRGRD

PCCRGRD

PERFUNC

PFCORR

PFCRAP

PFFFF

PFFNF

PFREIM

PFSET

PLTFILE

POLYN

POLYNXY

PPWSNF

PRDCTC2

PRDRTC2

PRDTC2

PRNCORR
MaKe a PoLYnomial function DZ of $\mathrm{X}$ and $\mathrm{Y}$

get the Minimum and Maximum of the Imaginary part of a Complex Array get the Minimum and Maximum of the Real part of a Complex Array create a complex array equal to a FUNCtion in $\mathrm{X}$ times a function in $\mathrm{Y}$ given a Near-Field, compute the corresponding Far-Field

MODulate a complex array with a function of $\mathrm{X}$

MODulate a complex array with a function of $\mathrm{Y}$

given a Near-Field, obtain the corresponding Psudo Far-Field

convert a complex array of R-I format to A-P format and OUTput the result as two ASCii data files

OUTput a Direct Access Complex Binary data file

OUTput a complex array to Disk storage, the Printout file, and/or a Storage array

setup to OUTput amplitude and phase of a complex array to a .GRD file get 4 file names and OUTput 4 arrays as .PLT files

OUTput 4 amplitude and phase arrays formed from a column and a row of a complex array as .PLT Files

OUTput a Real array to a .GRD file for input to 'surfer' graphics codes

for a Complex array, Print a Column and/or Row of amplitude and phase values and then output the amplitude and pahse data to two .GRD files

dummy routine to select the name of a FUNCtion to be evaluated

obtain a Column OR Row of data and store the real or imaginary part for submital to a Plot File

obtain one dim. Amp. and Phase arrays from a Column and a Row of a complex array for submital to a Plot File

given a Psudo Far-Field, obtain the corresponding Far-Field

given a Psudo Far-Field, obtain the corresponding Near-Field

obtain Real or Imaginary part of $1 \mathrm{dim}$. complex array and copy to 2nd column of a real 2 dim. array

Plot File SETup: collect column or row data from a complex array and convert to R-I format (or A-P format)

output a real .PLT file for PLoTing multiple two-dimensional curves

function call to sum a POLYNomial of a single variable

calculate an array equal to a POLYNomial function of $\mathrm{X}$ added to a polynomial function of $\mathrm{Y}$

given log of Plane-Wave Spectrum, calculate Near Field via direct sum

raise to a Power a Real Double precision Column array; then Times a Complex array: 2 dimensional result

raise to a Power a Real Double precision Row array; then Times a Complex array: 2 dimensional result

raise to a Power a Real 2 dimensional Double precision array: then Times a Complex array: 2 dimensional result

PRiNt out the amplitude and phase of a Column OR a Row of complex array 
PRiNt and output to 4 .PLT files the amp. and phase of a column and a row of a complex array

PRNRCOR

PRiNt out a Real-array's Column OR Row

PRNTC1D

PRiNT the maximum amplitude of a Complex array and one column of Data

PRNTR1D

PRiNT maximum and minimum values of a real array and one column of Data

RADDRC2 Real array ADDed to a Real Constant, 2 dimensional

RANGED

RANGES

RARYMM2

RCA2B1

RCBD2

RCBDIOF

RCBDPAR

RCBDSET

RDCBD1

RDCBD2

RDDABP

RDDACBD

RDIF2

RDOT

RDRBD2

REARANG

RINIT1

RMULT2

RNDM

RNDMDZ

RRA2B1

RZTORC1

RZTORC2

SCLCC1

SCLCC2

SCLRC1

SCLRR2

SETBNDR

SETFILS

SETFIOF

SETFPAR

SETTSZ

SFTCACB

lower and upper RANGE values of a Double precision 1 dimensional array lower and upper RANGE values of a Single precision 1 dimensional array get a Real ARraY's Maximum and Minimum values, 2 dimensional array copy a Real Column of data from array A 2 dim. to array B 1 dim.

Read in a Real binary dataset and store as a Complex Binary Dataset in a 2 dimensional array

get the Input Output File name for inputing 2 Real Binary data sets into a Complex array

Read PARameters and file names for inputing 2 Real Binary data sets into a Complex array

Set up to read two Real Binary data sets into a Complex array

Read in a Real Binary data set into a Complex 1 dimensional array

ReaD a Complex Binary data set into a 2 dimensional array

ReaD a Direct Access Binary data set for the set Parameters

ReaD a Direct Access Complex Binary Data set

get the Real Difference of two dimensional real arrays

for two Real arrays form their DOT product

ReaD a Real Binary data set into a 2 dimensional array

amplitude, phase, distance correction and swap to obtain far-field data

Real INITialization of 1 dimenstional array with a real constant

Real MULTiplication of real arrays in 2 dimensions

function call to return a RANDoM number

calculate a real array DZ all of whose elements are RANDoMly obtained

copy a Real Row of data from array A 2 dim. to array B $1 \mathrm{dim}$.

Raise a 1 dimensional array TO a Real Constant power

Raise a 2 dimensional array TO a Real Constant power

SCaLe by a Complex Constant a Complex array in 1 dimension

SCaLe by a Complex Constant a Complex array in 2 dimension

SCaLe by a Real Constant a Complex array in 1 dimension

SCaLe by a Real Constant a Real array in 1 dimension

SET the BouNDaRy regions of complex array CDATA to complex constant CC

SET up to read a list of FILES containing formated-output file names get the Input Output File for reading formated-data-output file names read the names of designated formated-data-output files SET up the necessary arrays for TayLoR series in $\mathrm{Z}$ calculations ShiFT the location of Complex data in zero padded array to array center 
SFTRARB

SIN4X

SWAP

TIMER

TIMERS

TODAY

TSZK

TSZK1

TSZSLM

TSZSLM0

TSZSLM1

UDASCUN

UDDSIUN

UDDZIUN

UDFFIUN

UDFIUN

UDNFIUN

WCBD1

WCBD2

WDACBD

WLTOCM

WRBD2

WRCHIF

XCHAR

XSCHAR

XYGRIDS
ShiFT the location of Real data in zero padded array to array center calculate the function SINe of X raised to the fourth power

SWitch begining to end Array-element Positions of both rows and columns store system TIME on first call, return time difference on second call multiple TIME initilizations, time differences returned on second call write current date to screen add TayLoR Series (in Z) term of order $\mathrm{K}$ to existing CDATA array obtain sum of Kth Taylor Series term with CDATA then output partial sum Taylor Series in Z Summation from Low order to Max order added to CDATA initialize Taylor Series in Z with terms Summed from Low to Max order Taylor Series in Z Sum from Low to Max order, each partial sum output UpDate file record of ASCii output-file UNits UpDate Direct-Sum Integer-UNit-number file of output-name extensions UpDate file record of DZ file-name extensions that have been created UpDate file record of existing Far-Field file-name extensions UpDate File-Index Unit Number file

UpDate file record of existing Near-Field file-name extensions

Write an unformatted Complex Binary Data set, which is 1 dimensional Write an unformatted Complex Binary Data set, which is 2 dimensional Write a Direct Access Complex Binary Dataset which is self-documented given a frequency, convert from Wave-Lengths to CentiMeters Write an unformatted Real Binary Data set, which is 2 dimensional WRite a CHecK list of parameters to the standard print file eXpress an integer modulus 100 as a CHARacter variable eXpress an integer modulus 10 as a Single CHARacter variable set up both X and Y GRIDs using Single precision 


\section{Appendix A}

\section{Creating the Original Direct Access Binary Dataset}

Two modules are provided for inputing ascii data files to create direct-access complex binary datasets. The module UAPDACB reads in two ascii files, one containing amplitude data and one containing phase data. For these files, the data in each column precedes the data in each succeeding column. The required structure of these ascii files is seen by inspection of subroutine FRRAD or subroutine FRRADHD (the latter assumes that a 120 character HeaDer preceedes the ascii data). Alternately, module UDBPDACB is used to read two ascii files, one containing amplitude data expressed in decibels and one containing phase data. Both files are assumed to have been set up as .GRD files suitable for input to the system plot package. For these files, the data in each row preceeds the data in each succeeding row. The structure of these ascii files is seen by inspection of subroutine FRGRD. The parameters associated with the binary datasets created by these modules are obtained from a user-supplied parameter file, which also contains the names of the two ascii input files, a format specification for the ascii input data, and the name of the binary output file. This user-supplied parameter file is specified in the file ADAB.IOF. The parameter file entries are seen by inspection of subroutine ADABPAR, which reads the file. The parameters occupying the first seven records of the direct-access binary file are defined in the following table:

\section{List of Parameters Included in the Direct Access Binary Datasets}

LENGTH the LENGTH of each record in the file, nominally equal to $8^{*} \mathrm{NY}$ (required for Direct Access files)

FFORNF data type specifier distinguishes between Far-Field OR Near-Field data

LABEL character variable of up to 120 characters identifying the file

NY, NX the number of respective columns and rows in the complex data array

DY, DX

FREQ

$\mathrm{ZO}$ incremental data point spacing in near-field plane for $\mathrm{Y}$ and $\mathrm{X}$ axes operating frequency expressed in gigahertz $\mathrm{z}$-axis distance in centimeters to the near-field measurement plane 


\section{System Initialization}

At the beginning of any research project the system has to be initialized to properly set the the system parameters and the far-field and near-field unit numbers. This is accomplished by executing the module UINITUN, which will write the following table to the screen:

THE INITIAL SETTINGS are:

$\begin{array}{rr}\text { ampordb.grd: ampordb }=: & \mathrm{dB} \\ \text { filter.ff: cksqrd }=: & 0.0000000 \mathrm{E}+00 \\ \text { order.drv: idrvinc,iorder }=: & 1 \\ \text { scale.dz: scalinc, dzscale=: } & 0.0000000 \mathrm{E}+00 \\ \text { fun.dz: funtype }=: & \text { per } \\ \text { active.iun: iactive }=: & 0 \\ \text { add.iun: iadd }=: & 0 \\ \text { amp2.iun: iunamp2=: } & 0 \\ \text { asci.iun: iunasci=: } & 7 \\ \text { difdiv.iun: idifdiv }=: & 1 \\ \text { dif2.iun: iundif }=: & 0 \\ \text { dif.iun: iundif }=: & 0 \\ \text { difdb.iun: iundfdb }=: & 0 \\ \text { div.iun: iundiv }=: & 0 \\ \text { drv.iun: iundrv }=: & 0 \\ \text { ds.iun: iunds } 0, \text { iundsl }=: & -1 \\ \text { dz.iun: iundz=: } & 61 \\ \text { ec.iun: iunec=: } & 0 \\ \text { err.iun: iunerr }=: & 0 \\ \text { ff.iun: iunff=: } & 60 \\ \text { nf.iun: iunnf }=: & 40 \\ \text { rdiv.iun: iunrdiv }=: & 0 \\ \text { ts.iun: iunts }=: & 0\end{array}$

STOP: UINITUN: normal termination

In the above table the first entries on each line give the name of the file where the information is recorded, the second entries give the name of the fortran variable(s) in the modules that contain the value(s), which are shown last. The key abbreviations in the file names and variable names can be deciphered by consulting Table 1. For example, iunasci specifies the current setting of the ascii output unit number, and iundz specifies the unit number of the $d z$ dataset. Many of the unit numbers are set to 0 , simply signifying that no data has yet been created for these fields. There are a few remaining variables included in the table that have special meanings. These 
are defined below:

ampordb .GRD files will be created in $\mathrm{dB}$; it can also be set to $a m p$

cksqrd filter limit for truncating plane-wave spectrum

idrivinc increment by which iorder is increased whenever order.drv is accessed by module UDERIV

iorder order of derivative calculated by module UDERIV

funtype TYPE of FUNction used by module UMAKEDZ to create incremental scalar field. It may have the value per (periodic), poly (polynomial), or ran (random) function

iunds0 initial value of iundsl: set to -1 when first initialized, thereafter equal to 0 iundsl single digit used as the last character in the filename extension, exclusively used by module UDS 


\section{Appendix C \\ System Status Reports}

After the execution of any module one can request a system status report to examine the system parameter settings and the unit number settings. This is accomplished by executing USHOWUN. One might do this to check the sequence of executions for correctness and to decide what data management steps one needs to take to access the next dataset needed to continue the research correctly. When USHOWUN is executed after URDNFFF and UMAKEDZ have been executed only once the following table is displayed:

THE CURRENT SETTINGS are:

\begin{tabular}{|c|c|c|}
\hline ampordb.grd: & $\mathrm{dB}$ & \\
\hline filter.ff: & $0.0000000 \mathrm{E}+00$ & \\
\hline order.drv: & 1 & 0 \\
\hline scale.dz: & $0.0000000 \mathrm{E}+00$ & 0.2000000 \\
\hline ampff,invff: & 1987.822 & $5.0306314 \mathrm{E}-04$ \\
\hline ampnf,invnf: & 1.059250 & $9.4406420 \mathrm{E}-01$ \\
\hline ffornf: & $\mathrm{ff}$ & \\
\hline fun.dz: & per & \\
\hline active.iun: & 0 & \\
\hline add.iun: & 0 & \\
\hline amp2.iun: & 0 & \\
\hline asci.iun: & 7 & 9 \\
\hline inc difdiv: & 1 & \\
\hline dif2.iun: & 0 & \\
\hline dif.iun: & 0 & \\
\hline difdb.iun: & 0 & \\
\hline div.iun: & 0 & \\
\hline drv.iun: & 0 & \\
\hline ds.iun: & -1 & 0 \\
\hline dz.iun: & 61 & 61 \\
\hline ec.iun: & 0 & \\
\hline err.iun: & 0 & \\
\hline ff.iun: & 60 & 60 \\
\hline nf.iun: & 40 & 40 \\
\hline rdiv.iun: & 0 & \\
\hline ts.iun: & 0 & \\
\hline
\end{tabular}

STOP: USHOWUN: unit status report complete 
Most of features and entries in the above table have been explained in Appendix A. Here, however, some of the entries show 2 unit numbers. The combinations of 2 equal unit numbers signifies that the modules writing these unit numbers have only been executed once, thereby making the initial unit numbers, as defined in Appendix A, the current unit numbers.

After creating all the datasets required by the error correction research problem (see Section 4), USHOWUN can be executed to get an overview of the system status. The output table appears as below:

\section{THE CURRENT SETTINGS are:}

$\begin{array}{rrr}\text { ampordb.grd: } & \mathrm{dB} & \\ \text { filter.ff: } & 0.0000000 \mathrm{E}+00 & 0 \\ \text { order.drv: } & 1 & 0.2000000 \\ \text { scale.dz: } & 0.0000000 \mathrm{E}+00 & \\ \text { ampff,invff: } & 1987.822 & 5.0306314 \mathrm{E}-04 \\ \text { ampnf,invnf: } & 1.059250 & 9.4406420 \mathrm{E}-01 \\ \text { ffornf: } & \mathrm{ff} & \\ \text { fun.dz: } & \mathrm{per} & \\ \text { active.iun: } & 59 & \\ \text { add.iun: } & 60 & \\ \text { amp2.iun: } & 0 & \\ \text { asci.iun: } & 7 & \\ \text { inc difdiv: } & 1 & \\ \text { dif2.iun: } & 0 & \\ \text { dif.iun: } & 0 & \\ \text { difdb.iun: } & 0 & \\ \text { div.iun: } & 0 & \\ \text { drv.iun: } & 0 & \\ \text { ds.iun: } & -1 & \\ \text { dz.iun: } & 61 & \\ \text { ec.iun: } & 42 & \\ \text { err.iun: } & 0 & \\ \text { ff.iun: } & 60 & \\ \text { nf.iun: } & 40 & \\ \text { rdiv.iun: } & 0 & \\ \text { ts.iun: } & 41 & \\ & & \end{array}$

STOP: USHOWUN: unit status report complete

Now we see that two unequal unit numbers appear in some of the entries. These indicate the range of unit numbers for the particular type of field, ( $f f$ or $n f$ ), that exist after repeated executions of the various modules. The first unit number indicates the initial unit number created and the last number indicates the current 
value of the unit number. The dataset referred to by the current value of the unit number will be automatically accessed if the value in active.iun is 0 . In addition, all special types of near fields that have been created during the course of the research are recorded in their respective unit number files. For example, the entry under ts.iun is 41 , meaning that the dataset with filename fort. 41 contains the errorcontaminated near field that was created using the Taylor series method. 


\section{Appendix D}

An index of PNFC subroutines and the calling sequences in these routines.

ACPCFFD

ACPCNFD

ADABIOF : chlngth

ADABPAR : errmess

ADDBOX

AMPDIF2

APDSET1

: cca2b1 cra2b1 criap1 capri1 cdif1

CABD

: cabdiof cabdpar frbw

CABDIOF

: chlngth

CABDPAR

: errmess

CADACB

: adabiof adabpar frradhd capri wdacbd

CADD1

CADD2

CAEIPH2

CAEIPHC

CAPCCD1

CAPRI

CAPRI1

CAPRNT

: mmrca prntrld mmica

CARAYMX2

CARCBD2

CATOCB2

CBDTODB

: rdcbd2 criap1 db1

CCA2B1

CDFSET1

: cca2b1 cra2b1 cdif1 criap1 capri1

CDIF1

CDIF2

CDIVDS2

CDOT

CGATHER

CHKEQFP

CHIKEQI

CHKLEQI

CHKPAR0

CHKPAR1

CHKPAR2

CHLNGTH

CIMGSTR

CINIT1

CINIT2 
CIRCFLT

CMULDS2

CMULRD2

CMULT1

CMULT2

CMULTR2

CNIMCC1

CNTCACB

CONST

: iyjxcnt sftcacb

CONSTAX

COS2

COS3

COS4

COSAX

COSAX2

COSX

CRA2B1

CRATIO2

CRIAP

CRIAP1

CRNFERR

: nfpff catocb2 ffnf

CSMWCP1

CSMWCP2

CSUM1

CSUM2

CSUMCP1

CSUMCP2

CSUMIK2

CSUMRW1

CSUMRW2

CSWCPE2

CXPCLOG
DABDIOF : chlngth
DABDPAR : chkpar0 chkpar1
DATORB1
DB1
DB2
DCLN2
DNFDX : catocb2 prdrtc2 sclec1 fourt acpenfd swap
DNFDY : catocb2 prdctc2 sclcc1 fourt acpenfd swap
DNFDZ : dnfdze cmulds2
DNFDZE : catocb2 prdrtc2 fourt acpenfd swap sclrc1
DNFDZO : migammz cmulds2 dnfdze 
DSPWS

ECEXP

EIGAMAZ

ERRMESS

FAXSBYS $\quad$ fx fy (unspecified functions)

FBTIOF

: chlngth

FBTPAR

FFNF

: ffpff pffnf

FFNFZXY

: dcln2 migammz ppwsnf sclcc2

FFPFF

: eigamaz fltlimt flteigz cmulds2

FFTFFT

: sclrr2 fourt

FILSIOF

FILSPAR

FINDEND

FLTEIGZ

FLTGAMA

FLTLIMT

FLTPWSG

FLTRHIK

: fitlimt

FRBW

FRGRD

FRRAD

FRRADHD

FUNAXBY

: fx fy (unspecified functions)

FUNCSCL

FUNCXY

: nfff ffnf

FWDCRA1

: fx fy (unspecified functions)

FWRAD1

FWRAD2

GAMMASQ

GETFILE

GETWN

GRDDACB : adabiof adabpar frgrd capri wdacbd

GRID

GRIDD

HSTDFDB : findend

HSTDIF : findend

HSTDIV : findend

HSTDRV : findend

HSTDS : findend 


\begin{tabular}{|c|c|}
\hline $\begin{array}{l}\text { HSTEC } \\
\text { HSTFFNF } \\
\text { HSTMKDZ } \\
\text { HSTNFFF } \\
\text { HSTTS } \\
\text { HSTUN0 } \\
\text { HSTUN1 }\end{array}$ & $\begin{array}{l}\text { : findend } \\
: \text { findend } \\
: \text { findend } \\
: \text { findend } \\
: \text { findend } \\
: \text { findend } \\
: \text { findend }\end{array}$ \\
\hline $\begin{array}{l}\text { INPDABP } \\
\text { INPDACB } \\
\text { INPDRVP } \\
\text { INPDZP } \\
\text { INPFBT } \\
\text { INPFFP } \\
\text { INPFFP0 } \\
\text { INPFILS } \\
\text { INPGRDP } \\
\text { INPNFP } \\
\text { INPNFP0 } \\
\text { INPRCBD } \\
\text { INPTSP } \\
\text { INSLOSS } \\
\text { INTNF3 } \\
\text { IUNIT } \\
\text { IYJXCNT }\end{array}$ & $\begin{array}{l}\text { : dabdiof dabdpar rddabp getwn wrchkf } \\
: \text { dabdiof dabdpar rddacbd getwn wrchkf } \\
: \text { dabdiof dabdpar rddabp } \\
: \text { dabdiof dabdpar rddabp } \\
: \text { fbtiof fbtpar rcbdset } \\
: \text { dabdiof dabdpar rddabp } \\
: \text { dabdiof dabdpar rddacbd } \\
: \text { filsiof filspar } \\
: \text { dabdiof dabdpar rddabp } \\
: \text { dabdiof dabdpar rddabp } \\
: \text { dabdiof dabdpar rddacbd } \\
: \text { rcbdiof rcbdpar rcbdset } \\
: \text { dabdiof dabdpar rddabp } \\
: \text { sclcc1 } \\
: \text { migammz fourt swap cdivds2 acpcffd acpcnfd }\end{array}$ \\
\hline $\begin{array}{l}\text { LAPLCAN } \\
\text { LNPPWS }\end{array}$ & $\begin{array}{l}: \text { nfpff dnfdze dnfdx cadd } 2 \text { dnfdy pffnf } \\
: \text { carcbd } 2 \text { flpwsg }\end{array}$ \\
\hline $\begin{array}{l}\text { MAKEDZ } \\
\text { MAKPFF } \\
\text { MDARB1 } \\
\text { MDNFDX } \\
\text { MDNFDY } \\
\text { MDNFDZ } \\
\text { MDNFDZE } \\
\text { MDNFDZO } \\
\text { MIGAMMZ } \\
\text { MKGAMMA } \\
\text { MKPERDZ } \\
\text { MKPLYDZ } \\
\text { MMICA } \\
\text { MMRCA }\end{array}$ & $\begin{array}{l}: \text { grid funaxby sclrr } 2 \\
: \text { cinit1 addbox nfpff } \\
: \text { datorb1 } \\
: \text { dnfdx } \\
: \text { dnfdy } \\
: \text { mdnfdze mdnfdzo } \\
: \text { dnfdze } \\
: \text { cmulds } 2 \text { mdnfdze }\end{array}$ \\
\hline
\end{tabular}


NF

NFFF

: nfpff pffff

NFMODX

NFMODY

NFPFF

: setbndr fourt swap acpcffd

OUTASC

: fwrad2

OUTDACB

: setfils wdacbd

OUTDPS

: prncorr catocb2 wcbd2

OUTGRD

: criap db1 cnimcc1 mmrca getfile outrgrd mmica

OUTPFS0

: getfile pltfile

OUTPFS1

: pfcrap outpfs0

OUTRGRD

PCCRGRD

: caraymx 2 prncorr outgrd

PERFUNC

: cosax 2 , etc.

PFCORR

: pfset pfreim

PFCRAP

: pfcorr pfreim

PFFFF

PFFNF

PFREIM

: migammz cxpclog ecexp

: fourt acpcnfd swap

PFSET

PLTFILE

: cca2b1 cra2b1 criap1 capri1

POLYN

POLYNXY

: polyn

PPWSNF

: lnppws dspws

PRDCTC2

PRDRTC2

PRDTC2

PRGTSZ

: catocb2 ffnf tszSLM0

PRNCORR

: cca2b1 criap1 cra2b1

PRNPLT

: prncorr outpfs1

PRNRCOR

: rca2b1 rra2b1

PRNTC1D

: caraymx2

PRNTRID

RADDRC2

RANGED

RANGES

RARYMM2

RCA2B1

RCBD2

: errmess

RCBDIOF

RCBDPAR

: chlngth 


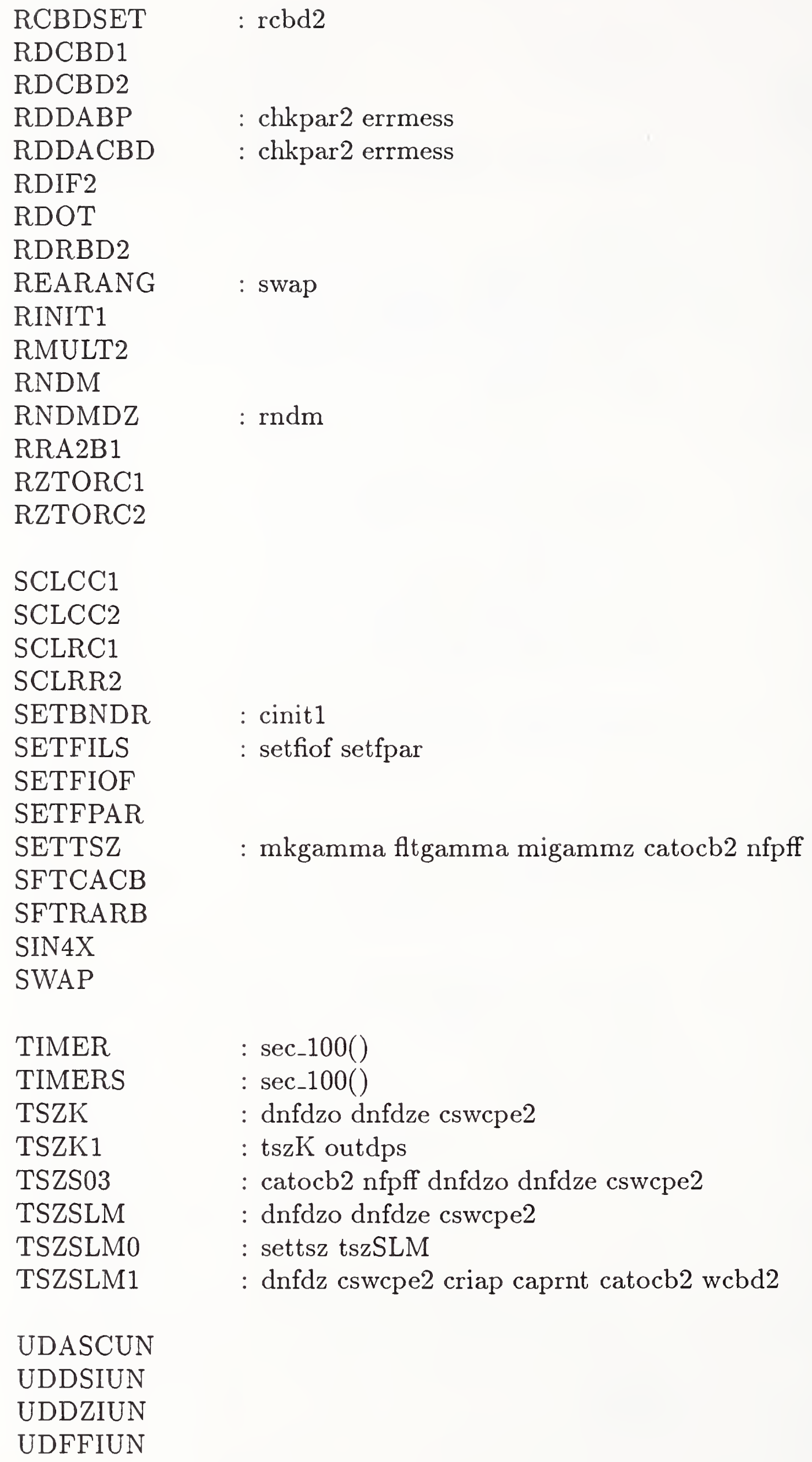




\section{UDFIUN}

UDNFIUN

WCBD1

WCBD2

WDACBD

WLTOCM

WRBD2

WRCHKF : wltocm

XCHAR

XSCHAR

XYGRIDS : grid 


\begin{tabular}{|c|c|c|}
\hline \multirow{3}{*}{$\begin{array}{l}\text { NIST-114A } \\
\text { (REV. 3-89) }\end{array}$} & \multirow{3}{*}{$\begin{array}{l}\text { U.S. DEPARTMENT OF COMMERCE } \\
\text { NATIONAL INSTITUTE OF STANDARDS AND TECHNOLOGY } \\
\text { BIBLIOGRAPHIC DATA SHEET }\end{array}$} & 1. PUBLICATION OR REPORT NUMBER \\
\hline & & PERFORMING ORGANIZATION REPORT NUMBEF \\
\hline & & 3. PUBLICATION DATE \\
\hline
\end{tabular}

4. TITLE AND SUBTITLE

Planar Near-Field Codes for Personal Computers

5. AUTHOR(S)

Lorant A. Muth and Richard L. Lewis

6. PERFORMING ORGANIZATION (IF JOINT OR OTHER THAN NIST, SEE INSTRUCTIONS)

U.S. DEPARTMENT OF COMMERCE

NATIONAL INSTITUTE OF STANDARDS AND TECHNOLOGY

GAITHERSBURG, MD 20899

7. CONTRACT/GRANT NUMBER

8. TYPE OF REPORT AND PERIOD COVERED

9. SPONSORING ORGANIZATION NAME AND COMPLETE ADDRESS (STREET, CITY, STATE, ZIP)

Sponsored, in part, by

Air Force Guidance and Metrology Center.

Newark Air Force Base, Ohio 43057

(Contract SD10-WPD-B238)

10. SUPPLEMENTARY NOTES

DOCUMENT DESCRIBES A COMPUTER PROGRAM; SF-185, FIPS SOFTWARE SUMMARY, IS ATTACHED.

11. ABSTRACT (A 200-WORD OR LESS FACTUAL SUMMARY OF MOST SIGNIFICANT INFORMATION. OF DOCUMENT INCLUDES A SIGNIFICANT BIBLIOGRAPHY OR LITERATURE SURVEY, MENTION IT HERE.)

We have developed planar near-field codes, written in Fortran, to serve as a research tool in antenna metrology. We describe some of the inner workings of the codes, the data management schemes, and the structure of the input/output sections to enable scientists and programmers to use these codes effectively. The structure of the codes is seen to be open, so that a user can incorporate a new application into the package for future use with relative ease. The large number of subroutines currently in existence are briefly described, and a table showing the interdependence among these subroutines is constructed. Some basic research problems, such as transformation of a near field to the far field and probe position error correction, are carried out from start to finish, to illustrate use and effectiveness of these codes. Sample outputs are shown. The advantage of a high degree of modularization is demonstrated by the use of DOS batch files to execute Fortran modules in a desired sequence.

12. KEY WORDS (6 TO 12 ENTRIES; ALPHABETICAL ORDER; CAPITALIZE ONLY PROPER NAMES; AND SEPARATE KEY WORDS BY SEMICOLONS)

antenna metrology; data management; planar near-field codes; research tool; subroutines

FOR OFFICIAL DISTRIBUTION. DO NOT RELEASE TO NATIONAL TECHNICAL INFORMATION SERVICE (NTIS).

14. NUMBER OF PRINTED PAGES

$$
52
$$

15. PRICE 

IR 89-3930

\section{CANCELLED}

\section{UNAVAILABLE FOR BINDING}



(2) 
